\title{
RETRACTED ARTICLE: Deeply fusing multimodal features in hypergraph
}

\author{
Wei Yao ${ }^{1} \cdot$ Ying Jiang $^{1} \cdot \mathrm{Jun} \mathrm{Chen}^{2} \cdot$ Linchao Xie $^{2}$ • \\ Caiyou Zhang ${ }^{1}$
}

Received: 9 October 2017 /Revised: 12 October 2017 / Accepted: 13 October 2017 /

Published online: 14 November 2017

(C) Springer Science+Business Media, LLC 2017

The Editor-in-Chief has retracted this article [1], which was published as part of special issue "Multi-source Weak Data Management using Big Data", because there is evidence suggesting authorship manipulation and an attempt to subvert the peer review process.

The authors have not responded to correspondence about this retraction.

\section{References}

1. Yao, W., Jiang, Y., Chen, J. et al. Deeply fusing multimodal features in hypergraph. Multimed Tools Appl (2017). https://doi.org/10.1007/s11042-017-5305-6

Electronic supplementary material The online version of this article (https://doi.org/10.1007/s11042-0175305-6) contains supplementary material, which is available to authorized users.

Caiyou Zhang

zhangcaiyou1778@126.com

1 State Grid Zhejiang Electric Power Company Information \& Telecommunication Branch, Hangzhou, China

2 Zhejiang Huayun Electric Power Industrial Group, Wenzhou, China 